\title{
Polygenic risk score analyses of symptoms and treatment response in an antipsychotic-naive first episode of psychosis cohort
}

Marcos Leite Santoro ${ }^{1,2}$, Vanessa Ota ${ }^{1,2}$, Simone de Jong ${ }^{3}$, Cristiano Noto ${ }^{4,5}$, Leticia M. Spindola ${ }^{2,4}$, Fernanda Talarico ${ }^{2,4}$, Eduardo Gouvea ${ }^{4,5}$, Sang Hyuck Lee ${ }^{3,6}$, Patricia Moretti ${ }^{2,4}$, Charles Curtis ${ }^{3,6}$, Hamel Patel ${ }^{3,6}$, Stephen Newhouse (1) ${ }^{3,6}$, Carolina Muniz Carvalho ${ }^{2,4}$, Ary Gadelha ${ }^{2,4}$, Quirino Cordeiro ${ }^{4,5}$, Rodrigo Affonseca Bressan ${ }^{2,4}$,

Sintia lole Belangero ${ }^{1,2,4}$ and Gerome Breen ${ }^{3,6}$

\begin{abstract}
In this study, we aimed to test if the schizophrenia (SCZ) polygenic risk score (PRS) was associated with clinical symptoms in (a) the first episode of psychosis pre-treatment (FEP), (b) at nine weeks after initiation of risperidone treatment (FEP-9W) and (c) with the response to risperidone. We performed a detailed clinical assessment of 60 FEP patients who were antipsychotic-naive and, again, after nine weeks of standardized treatment with risperidone. After blood collection and DNA isolation, the samples were genotyped using the Illumina PsychArrayChip and then imputed. To calculate PRS, we used the latest available GWAS summary statistics from the Psychiatric Genomics Consortium wave-2 SCZ group as a training set. We used Poisson regression to test association between PRS and clinical measurements correcting for the four principal components (genotyping). We considered a p-value $<0.0014$ (Bonferroni correction) as significant. First, we verified that the schizophrenia PRS was also able to distinguish cases from controls in this south-eastern Brazilian sample, with a similar variance explained to that seen in Northern European populations. In addition, within-cases analyses, we found that PRS is significantly correlated with baseline (pre-treatment) symptoms, as measured by lower clinical global assessment of functioning (-GAF), higher depressive symptoms and higher scores on a derived excitement factor. After standardized treatment for nine weeks, the correlation with GAF and the excitement factor disappeared while depressive symptoms became negatively associated with PRS. We conclude that drug (and other treatments) may confound attempts to understand the aetiological influence on symptomatology of polygenic risk scores. These results highlight the importance of studying schizophrenia, and other disorders, pre-treatment to understand the relationship between polygenic risk and phenotypic features.
\end{abstract}

Correspondence: Gerome Breen (gerome.breen@gmail.com)

'Genetics Division, Federal University of São Paulo (UNIFESP), São Paulo, SP, Brazil

${ }^{2}$ Interdisciplinary Laboratory of Clinical Neurosciences, Federal University of São Paulo (UNIFESP), São Paulo, SP, Brazil

Full list of author information is available at the end of the article.

\section{Introduction}

Schizophrenia (SCZ) is a severe mental disorder affecting $\sim 1 \%$ of the population and is characterized by the presence of psychosis and other features, such as negative (i.e., flattened affect and social withdrawal) and disorganization symptoms (e.g., disorganized speech and behaviour). Symptomatic and psychosocial deterioration progress rapidly during the period just after the onset of

\section{(c) The Author(s) 2018}

(c) Open Access This article is licensed under a Creative Commons Attribution 4.0 International License, which permits use, sharing, adaptation, distribution and reproduction cc) in any medium or format, as long as you give appropriate credit to the original author(s) and the source, provide a link to the Creative Commons license, and indicate if changes were made. The images or other third party material in this article are included in the article's Creative Commons license, unless indicated otherwise in a credit line to the material. If material is not included in the article's Creative Commons license and your intended use is not permitted by statutory regulation or exceeds the permitted use, you will need to obtain permission directly from the copyright holder. To view a copy of this license, visit http://creativecommons.org/licenses/by/4.0/. 
the disorder, termed the first episode of psychosis (FEP) ${ }^{1}$. Moreover, reports indicate that brain abnormalities and cognitive deficits are already present in the $\mathrm{FEP}^{1}$, even though patients are not affected yet long exposure to antipsychotics ${ }^{2,3}$.

SCZ is highly heritable $(\sim 80 \%)^{4}$. The most recent Genome-Wide Association Study (GWAS) for SCZ in the Psychiatric Genomics Consortium (PGC) wave 2 (PGC2) tested the association of millions of single-nucleotide polymorphisms (SNPs) and other types of genetic variations in $\sim 34,000$ cases and $\sim 113,000$ controls, and was particularly successful in uncovering new genes and pathways for the disorder ${ }^{5}$. For SCZ (and other psychiatric disorders), it is now well accepted that, while no single variant accounts for a large proportion of cases, thousands of genetic variants act together to confer the majority of the genetic risk for the disorder-a polygenic architecture of risk ${ }^{6,7}$.

Purcell et al. ${ }^{8}$ developed and applied the method proposed by Wray et al. ${ }^{7}$ to calculate a polygenic risk score (PRS) explaining 2-3\% of variance in SCZ case-control status $^{7,8}$. With the large increases in sample size enabled by international GWAS consortia, the SCZ PRS has become more powerful. Using the PGC2 SCZ GWAS as a training sample ${ }^{5}$, predictive SNPs achieving a nominal $p$ value threshold can be selected and the PRS of an individual in independent sample can be calculated. More specifically, the effect size estimated in the training sample for each SNP's risk allele is multiplied by the number of risk alleles present in an individual. This is then summed across all variants selected in the genome to yield a PRS for every individual in a training sample ${ }^{8,9}$.

Such a PRS has a much larger effect size than any single genetic variant and does not need a large sample size for the target sample as long as it is estimated from a very large training sample ${ }^{9}$. The SCZ PRS represents a genetic estimate of liability to the disorder and is a normally distributed quantitative trait that can be applied in many ways. For SCZ, the PRS has been correlated with quantitative variables, such as severity of symptoms ${ }^{10}$ and prefrontal activity ${ }^{11}$. For bipolar disorder, PRS has been correlated to function and brain structures in individuals at risk ${ }^{12,13}$, and, for depression, it has been correlated with the reduction of the cortical volume in specific regions ${ }^{14}$. A recent paper by Vassos et al. ${ }^{15}$ reported that SCZ PRS is associated with diagnosis inFEP patients. One recent study reported a positive correlation between SCZ PRS and negative symptoms in an (unaffected) adolescent population cohort $^{16}$. Recently, another article found positive associations between a genetic (rather than polygenic) risk score generated with 84 SNPs with positive and negative symptoms at the FEP, but not after treatment ${ }^{17}$.
No study, to our knowledge, has examined the correlation of PRS with the untreated symptom profile of SCZ patients or the response to treatment in FEP patients. In this study, we test if the SCZ PRS is correlated with symptomatology, severity and response to antipsychotics during FEP in a serial longitudinal sample of initially treatment naive patients.

\section{Methods}

\section{Recruitment and consent}

We enrolled patients with FEP at admission to the Centro de Atenção Integral a Saúde Mental (CAISM), São Paulo. The study protocol was designed to address the acute but temporary lack of capacity in FEP patients at admission. When a patient was admitted meeting the inclusion criteria (below), medical staff explained the study to family members, provided printed information sheets and, if agreeing, families then signed a written informed consultee consent with the assent of the patient. At the follow-up assessment, the patients were directly consented into the study, provided they had capacity. If subjects lacked capacity at the follow-up assessment, consent was taken at a later stage when capacity was regained. The local Research Ethics Committee of Universidade Federal de São Paulo (CEP-UNIFESP 0603/10) and the national Brazilian Ethics Committee (CONEPCAAE 33148114.6.0000.5505, CAAE 48242015.9.0000.5505) approved the research protocol.

\section{Longitudinal cohort of FEP patients}

Our cohort of antipsychotic-naiveFEP patients includes 154 subjects recruited from a psychiatric emergency unit in São Paulo (Brazil). The diagnosis of a psychotic disorder was established by trained psychiatrists using Diagnostic and Statistical Manual of Mental Disorders, Fourth Edition (DSM-IV) criteria, using the Structured Clinical Interview for DSM-IV (SCID-I). Inclusion criteria were aged between 16 and 40 years without previous history of antipsychotic medication and with confirmed non-affective psychosis (SCZ, schizophreniform disorder or brief psychosis disorder diagnosis) after two months of treatment. Prior or current treatment with benzodiazepines was allowed. Patients with psychotic episodes due to a general medical condition, substance-induced psychotic disorder, intellectual disability, major depressive disorder or bipolar disorder were excluded.

A total of 60 patients met criteria for antipsychoticnaive FEP after the follow-up (FEP, $N=60$ ). These patients were assessed at baseline and followed up for $9.03 \pm 2.76$ weeks of risperidone treatment. Four patients were taking benzodiazepines and one clonazepam, at baseline. During follow-up, besides risperidone, 12 were taking clonazepam and 7 mood stabilizers. 
The healthy control group $(N=60)$ comprised agegender-and-ethnicity-matched volunteers with no firstdegree family history of psychotic disorders, who were evaluated by trained psychiatrists using a modified SCID-I to ensure no current or previous psychiatric diagnoses. Peripheral blood samples were collected in EDTA tubes at baseline and follow-up for patients and after psychiatric interview for controls.

\section{Clinical assessments}

All psychiatrists had the same training at the "Programa de Esquizofrenia da UNIFESP" and the FEP patients were always assessed by the same psychiatrist at both time points for the following scales: (a) PANSS (Positive and Negative Syndrome Scale), (b) CGI (Clinical Global Impression Scale) ${ }^{18}$, (c) GAF (Global Assessment of Functioning Scale), (d) CDSS (Calgary Depression Scale for $\mathrm{SCZ})^{19}$.

Symptom clusters (negative, positive, disorganization, excited and anxiety/depression) from the PANSS items ${ }^{20}$ were calculated using the algorithm from a previous study in a Brazilian population ${ }^{21}$. For more information about each symptom cluster, see Supplementary Table S1. Response to treatment was defined as a $>50 \%$ reduction in baseline PANSS total score ${ }^{22}$. GAF is the only scale where higher values represent less impairment; thus we transformed to them to negative values (referred to as $-\mathrm{GAF})$.

\section{DNA isolation}

Whole blood was collected into EDTA tubes, and genomic DNA isolation was performed using the Gentra Puregene Kit (Qiagen) according to the manufacturer's protocol.

\section{Genomic arrays}

The genotyping was performed at King's College London using the Infinium PsychArray-24 BeadChip (Illumina) with a GWAS core backbone ( $\sim 590 \mathrm{~K}$ markers) and specific content from the Psychiatric Genomics Consortium: https://www.med.unc.edu/pgc/psychchip.

\section{Quality control and imputation}

For the quality control (QC) parameters, we removed SNPs with a minor allele frequency $(\mathrm{MAF})<1 \%$, Locus missingness $>10 \%$ or Hardy-Weinberg disequilibrium significance $<0.00001$. We also excluded individuals with missingness $>10 \%$ and an estimation of identity-by-descent $>0.12$. Genotype imputation was performed using the https:/imputation.sanger.ac.uk using as Reference Panel the Haplotype Reference Consortium (release 1) with 32,488 samples (39 M sites) and the Pre-phasing algorithm SHAPEIT2. After post-imputation QC, using the same parameters as above, $\sim 9 \mathrm{M} \mathrm{SNPs}$ were analysed.

\section{Polygenic risk scores}

For more information about how the scores are calculated, please see the Supplementary Material of Purcell et $\mathrm{al}^{8}{ }^{8}$. To generate the PRS we used the PRSice software (www.PRSice.info) default options. The SCZ sample from PGC2 (downloaded from https://www.med.unc.edu/pgc) was used as the training sample and our imputed genotyping sample as the target. The PGC2 SCZ PRS is generated from many individual samples that may represent more chronic and severe SCZ, such as patients on clozapine. This means the PGC PRS represents a powerful tool to understand the influence of SCZ risk on clinically important symptom dimensions pre-treatment. We performed $P$-value-informed clumping with a cutoff of $r^{2}=$ 0.1 using a $250-\mathrm{kb}$ window and calculated scores per individual for multiple $p$-threshold (ranging from 0.0001 to 0.5 with increments of 0.00005 ) including or excluding the MHC (major histocompatibility complex) region on chromosome 6, which has a complex linkage disequilibrium structure. Given that our sample is sampled from an admixed south eastern Brazilian population, we carefully assessed population stratification and used the first four components generated by plink 1.9 software were used as covariables. Posteriorly, PRSice runs a regression to find the best $p$-threshold based on the explained variance (Nagelkerke's pseudo- $r^{2}$ correlation) and in our case gave PRSs based on the most FEP case-control variance explained.

\section{Statistical analysis}

We used R for all statistical analysis. With the PRSs calculated for the case-control comparison, we used a generalized linear model to test PRS associations assuming a Poisson distribution (Poisson regression), which is more suitable for ordinal variables (such as psychiatric scales), using clinical traits as the dependent variable and the best $p$-threshold PRS with the first four principal components as the independent variables and covariates. As clinical outcome variables, we considered, for both time points, GAF score, total CGI score, total PANSS scores and the five PANSS dimension clusters suggested by Wallwork et al. ${ }^{20}$ and validated by Higuchi et al. ${ }^{21}$ in the Brazilian population. GAF values were transformed to negative values (-GAF), so all clinical variables were easily compared, with high values meaning high symptomatology. We defined as outliers those observations lying beyond 1.5 times the 'Inter Quartile Range' - the difference between 75th and 25th quartiles.

We applied the Bonferroni correction for multiple comparisons (number of psychiatric scales tested $N=36$ ), considering as significant a $p$-value $<0.0014(0.05 / 36)$. As the Brazilian population is known to be a highly admixture population, we first plotted case and controls principal components to check if they have similar 
Table 1 Clinical and demographic characteristics of the participants in this study

\begin{tabular}{|c|c|c|c|c|}
\hline Variable & $\begin{array}{l}\text { Healthy controls } \\
(N=59)\end{array}$ & Antipsychotic-naive FEP $(N=60)$ & $\begin{array}{l}\text { FEP after treatment } \\
(N=60)\end{array}$ & $p$-value \\
\hline Gender (\%) & M:34 (57.6\%) & M:40 (66.7\%) & & 0.309 \\
\hline Age in years; mean (SD) & $25.97(7.48)$ & $25.63(7.46)$ & & 0.808 \\
\hline Currently smoking (\%) & $N=2(3.6 \%)$ & $N=12(23.5 \%)$ & & 0.002 \\
\hline Family history of psychosis (\%) & & $N=23(50 \%)$ & & \\
\hline Cannabis use (\%) & & $N=18(52.9 \%)$ & & \\
\hline Other drugs use (\%)* & & $N=13(41.9 \%)$ & & \\
\hline Family income in US\$/month (SD) & & $870.59(792.31)$ & & \\
\hline BMl in $\mathrm{kg} / \mathrm{m}^{2}$ & & $23.58(3.78)$ & & \\
\hline PANSS negative; mean (SD) & & $27.37(10.51)$ & $25.02(9.28)$ & 0.127 \\
\hline $\begin{array}{l}\text { PANSS disorganization/cognition; mean } \\
\text { (SD) }\end{array}$ & & $26.96(8.58)$ & $19.91(6.22)$ & $5.029 \times 10^{-8}$ \\
\hline PANSS excitement; mean (SD) & & $24.69(9.09)$ & $13.22(5.62)$ & $7.84 \times 10^{-14}$ \\
\hline PANSS positive; mean (SD) & & $34.75(7.32)$ & $21.23(9.52)$ & $2.46 \times 10^{-13}$ \\
\hline PANSS depression/anxiety; mean (SD) & & $24.24(8.79)$ & $18.11(7.85)$ & $2.97 \times 10^{-5}$ \\
\hline PANSS total & & 94.55 (20.94) & $68.21(20.31)$ & $1.71 \times 10^{-10}$ \\
\hline GAF; mean (SD) & & $31.21(10.52)$ & $55.47(16.61)$ & $1.34 \times 10^{-11}$ \\
\hline CGl; mean (SD) & & $4.83(0.72)$ & $3.35(1.26)$ & $1.77 \times 10^{-11}$ \\
\hline CDSS; mean (SD) & & $4.64(5.04)$ & $2.48(4.27)$ & 0.007 \\
\hline
\end{tabular}

M male, SD standard deviation, FEP first-episode psychosis, PANSS Positive and Negative Syndrome Scale, CGI Clinical Global Impression Scale, GAF Global Assessment of Functioning Scale, CDSS Calgary Depression Scale for Schizophrenia

*Drugs including cocaine, sedatives, stimulants, hallucinogens, opioids and gases

background and then we did a sensitivity analysis considering only full European ancestry cases.

Using the residuals from the PRS with principal components, we tested if the available demographics could be potential confounders. Further, we tested if response to risperidone overall or within subtypes of FEP included in our study (SCZ or schizophreniform) was associated with SCZ PRS. First, we tested the change in symptoms from baseline to the follow-up and if the subtype of FEP was associated with the PRS using a Poisson regression. Second, we tested the correlation between the change in total PANSS and PRS using a Pearson correlation. Finally, we verified if there was an association of clonazepam or mood stabilizers with CDSS, CGI, GAF and PANSS symptoms that could be affecting the results.

\section{Results}

Table 1 shows the clinical and demographic characteristics of the participants. Smoking rates significantly higher in cases than in controls. Patients showed improvement after nine weeks of risperidone treatment for all scales and symptom clusters, except for PANSS negative. Figure S8 shows a heatmap of the correlations among the tested clinical variables, demonstrating, a high correlation between PANSS depressive factor and CDSS, and between GAF and most clinical variables. Table S2 and S3 shows that there are no associations between demographics with either PRS or clinical variables.

\section{Brazilian admixture sample}

Cases and controls showed similar principal components structure, and when analysing only the European ancestry individuals (self-declared and consistent with the genetic estimates) the direction and magnitude of associations remained the same (Table S5 and Figures S1, S7).

\section{Polygenic risk}

The number of independent SNPs analysed for each threshold and cohort is described in Supplementary Table S4. With or without the MHC region, results were similar; thus, we carried on including the $\mathrm{MHC}$ region to increase the number of analysed SNPs and, consequently, the power of our analysis. The PRS was significantly different between cases and controls (Fig. 1) with a best $p$-threshold of $0.0112\left(N_{\mathrm{SNPs}}=21,622\right)$ and an explained variance of 0.19 (Nagelkerk's pseudo- $r^{2}$ ). Figure S7 shows the normal 


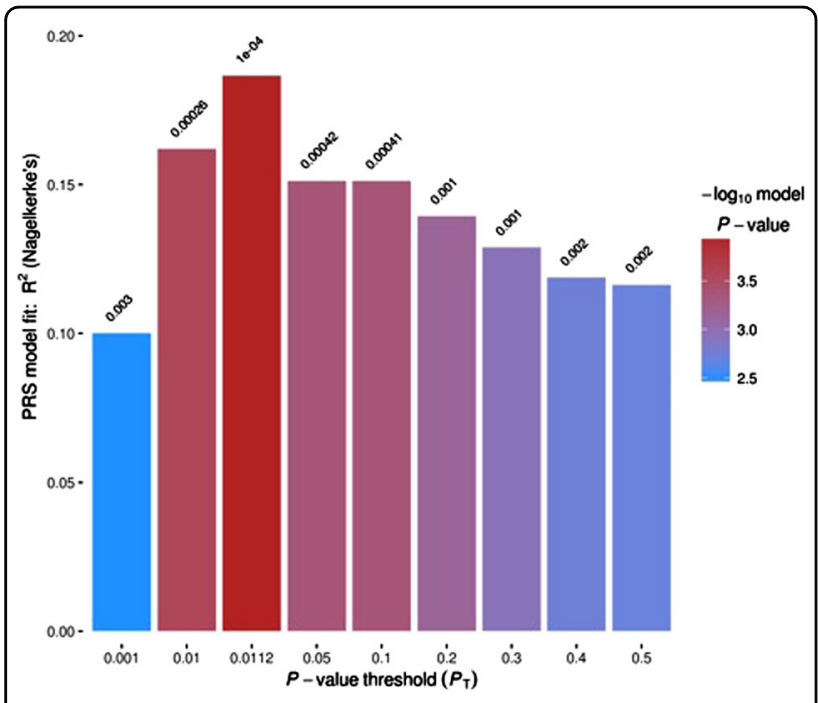

Fig. 1 Graph from PRSice showing the explained variance ( $y$-axis) for each $p$-threshold ( $x$-axis) to identify cases and controls for our sample

distribution of the PRS for cases and controls, and Figure S10 shows the odds ratio (OR) of psychosis for quantiles of PRS.

\section{PRS and clinical variables relation}

At baseline (antipsychotic naive FEP), we find a positive association PRS with the PANSS excitement factor (fivefactor model $)(B=566.7 ; p$-value $=0.0003)$ and a trend for association with -GAF $(B=436.1 ; p$-value $=0.003)$. PRS showed a positively trend for depressive symptoms at baseline (CDSS total: $B=1042.3$; $p$-value $=0.0039$ ) but became significantly negatively associated with depressive symptoms after risperidone treatment (CDSS total: $B=$ $-1800.2 ; p$-value $=0.0004)$. The results are summarized in Table 2 and Figures S2-S4.

Looking at response to risperidone, we analysed the five PANSS factors and other scales, we observed a positive association for $\triangle$ CDSS $(B=717 ; p$-value $=0.0006)$ (Table 3). However, considering the total PANSS, there was no correlation between $\Delta$ total PANSS and PRS $(t=0 ; 62633$, $\mathrm{df}=49 ; p$-value $=0.534 ; r=0.089)$. Although a borderline association was observed between PANSS-excitement at the follow-up and mood stabilizer use $(N=7)$, the relationship between PRS and PANSS-excitement remained not significant even adding this as a covariate $(B=-110, p=0.94)$.

Within FEP subtypes, depressive symptoms (CDSS) were positively associated with PRS in both FEP subtypes when analysed separately at baseline $\left(B_{\text {schizophrenia }}=\right.$ 1746.1; $\quad$ pschizophrenia $_{\text {s. }}=0.002 ; \quad B_{\text {schizophreniform }}=2660.0$; $\mathrm{p}_{\text {schizophreniform }}=0.036$ ), while PANSS excitement and -GAF was associated only in the schizophreniform subgroup $\left(B=1393.3 ; p=9 \times 10^{-5}\right.$ and $B=-1449.1 ; p=$
$1.3 \times 10^{-4}$, respectively) (Table 4, Supplementary Figs S5 and S6).

Given the different pattern of association between depressive symptoms (CDSS) and PRS at the baseline (positive association) and after treatment (negative association), we generated a trajectory plot to visualize each individual symptom in both time points (Figure S9). We can note that those individuals with high PRS tend to show a decrease in their depressive symptoms after risperidone treatment, while those with low PRS tend to maintain or increase their level of depressive symptoms.

\section{Discussion}

In this study, we demonstrate for the first time that the SCZ PRS is associated with different clinical symptoms during the pre-treatment stage of FEP. Although recently Sengupta et al. ${ }^{17}$ reported positive associations between PRS and clinical variables and FEP, it is important to note that our methods were different in many aspects; we included (1) only non-affective FEP, (2) only antipsychotic naive FEP, (3) applied a standardized treatment (risperidone) and (4) calculated the PRS using more than $21 \mathrm{~K}$ SNPs (compared with 84 used by them). Specifically, in pre-treatment FEP patients, we identified a positive correlation of PRS with depressive symptoms (CDSS total), excitement symptoms (PANSS-excitement factor) and with Global Assessment of Functioning (-GAF). After standardized treatment for 9 weeks with risperidone, we observed no positive association for these or other clinical measurements, but a negative correlation with PRS emerges for both CDSS and PANSS depressive/anxiety factor. Concordant with this, Sengupta et al. ${ }^{17}$ observed similar results for CDSS, although not reaching statistical significance, probably because of the lower power of including only 84 SNPs in the genetic risk score. These results suggest the potential aetiological importance of depression (and anxiety) in SCZ. In sensitivity analyses, we found that observed baseline positive correlation with PANSS excitement and -GAF was driven by the subgroup composed by individuals with schizophreniform or brief psychosis disorder diagnoses (Table 4), while the positive correlation of SCZ PRS with depressive symptoms was present irrespective of diagnostic group.

Looking at treatment response, we found that pretreatment baseline to post-treatment follow-up changes for PANSS excitement ( $\triangle$ PANSS excitement) and CDSS $(\triangle \mathrm{CDSS})$ were also positively correlated with SCZ PRS (Table 3), suggesting that patients with a higher PRS tend to show more improvement in symptoms after treatment (Figure S9) and that those with lower PRS have increased depressive symptoms. It is well known that some patients may have an increase in depressive symptoms once positive symptoms remit, being recognised as a clinical disorder (ICD F20.4 = post-SCZ depression). However, 
Table 2 PRS correlation with clinical variable during the baseline and the follow-up

\begin{tabular}{|c|c|c|c|c|c|}
\hline Time & Clinical variable & & $N$ & B & $p$-value \\
\hline \multirow[t]{12}{*}{ Baseline (antipsychotic naive FEP) } & $\mathrm{CGl}$ & & 50 & 72.8 & 0.8436 \\
\hline & ${ }^{a}-G A F$ & & 48 & 436.1 & 0.0030 \\
\hline & ${ }^{\mathrm{a}} \mathrm{CDSS}$ total & & 51 & 1042.3 & 0.0039 \\
\hline & PANSS total & & 53 & 38.1 & 0.6390 \\
\hline & aPANSS positive & & 53 & 400.0 & 0.0278 \\
\hline & PANSS negative & & 53 & -205.6 & 0.3048 \\
\hline & PANSS general psychopathology & & 53 & -20.2 & 0.8886 \\
\hline & Five-factor model $^{21}$ & PANSS negative & 53 & -168.2 & 0.2655 \\
\hline & & PANSS disorganization/cognition & 53 & -32.9 & 0.8281 \\
\hline & & bPANSS excitement & 53 & 566.7 & 0.0003 \\
\hline & & PANSS positive & 53 & 27.1 & 0.8382 \\
\hline & & PANSS depression/anxiety & 53 & -112.8 & 0.4761 \\
\hline \multirow[t]{12}{*}{ Follow-up (9 weeks treated with risperidone) } & $\mathrm{CGl}$ & & 51 & -137.4 & 0.7588 \\
\hline & $-\mathrm{GAF}$ & & 53 & -132.8 & 0.2281 \\
\hline & ${ }^{\mathrm{b}} \mathrm{CDSS}$ total & & 53 & -1800.2 & 0.0004 \\
\hline & PANSS total & & 54 & -113.4 & 0.2215 \\
\hline & PANSS positive & & 56 & 277.3 & 0.3141 \\
\hline & PANSS negative & & 56 & -358.3 & 0.0894 \\
\hline & PANSS general psychopathology & & 54 & -287.9 & 0.1140 \\
\hline & Five-factor model ${ }^{21}$ & PANSS negative & 56 & -180.6 & 0.2329 \\
\hline & & PANSS disorganization/cognition & 56 & -75.8 & 0.6608 \\
\hline & & PANSS excitement & 56 & 216.4 & 0.3048 \\
\hline & & PANSS positive & 56 & 10.0 & 0.9522 \\
\hline & & bPANSS depression/anxiety & 55 & -575.0 & 0.0013 \\
\hline
\end{tabular}

PANSS Positive and Negative Syndrome Scale, CGI Clinical Global Impression Scale, GAF Global Assessment of Functioning Scale, CDSS Calgary Depression Scale for Schizophrenia

${ }^{a}$ Significant $p$-values without Bonferroni correction

${ }^{\mathrm{b}}$ Significant $p$-values with Bonferroni correction

Table 3 PRS association with clinical variables of risperidone treatment response

\begin{tabular}{lllll}
\hline Clinical variables (follow-up - baseline) & N & B & p-value \\
\hline Five-factor model $^{21}$ & $\triangle$ PANSS negative & 53 & -71.6 & 0.5948 \\
& $\triangle$ PANSS positive & 53 & -74.5 & 0.6824 \\
& $\triangle$ PANSS disorganization & 53 & -35.3 & 0.8493 \\
& $\triangle$ PANSS depression/anxiety & 52 & 222.7 & 0.1173 \\
& ${ }^{a} \triangle$ PANSS excitement & 53 & 473.5 & 0.0034 \\
& & 49 & 717.2 & 0.0006 \\
\hline
\end{tabular}

Delta was calculated subtracting the measures of follow-up minus the baseline ${ }^{a}$ Significant $p$-values without Bonferroni correction

bignificant $p$-values with Bonferroni correction no study, to our knowledge has yet evaluated the relation between PRS and post-SCZ depression. Lastly, it is important to note that although we detected differences in these two symptom dimensions, we do not observe an association between total PANSS improvement ( $\triangle$ PANSS total) and PRS SCZ. Taken together, these results suggest that FEP patients who present with higher depressive and excitement symptoms and/or who show reduction in these dimensions after treatment with risperidone have a significantly higher genetic risk for SCZ (as estimated by PRS).

It is important to note that all previous studies of PRS and symptoms in SCZ used different study designs and, moreover, their samples were composed of patients under antipsychotic treatment. Vassos et al. ${ }^{15}$ suggested that the different subgroups in theirFEP sample (specifically non- 
Table 4 Association between the statistically significant clinical variables with PRS splitting the FEP patients into FEP subtypes according to the follow-up diagnosis

\begin{tabular}{|c|c|c|c|c|c|}
\hline Subtype & Clinical variable & Timepoint & $N$ & B & $p$-value \\
\hline \multirow[t]{7}{*}{ Schizophreniform } & a-GAF & Baseline & 9 & -1449.1 & 0.00013 \\
\hline & ${ }^{\mathrm{b}} \mathrm{CDSS}$ & Baseline & 9 & 2660.0 & 0.03594 \\
\hline & CDSS & Follow-up & 10 & -2323.7 & 0.20033 \\
\hline & aPANSS excitement & Baseline & 11 & 1393.3 & 0.00009 \\
\hline & PANSS depression/anxiety & Follow-up & 10 & -622.3 & 0.15126 \\
\hline & ${ }^{\mathrm{b}} \triangle \mathrm{PANSS}$ excitement & Baseline - follow-up & 11 & 877.8 & 0.02002 \\
\hline & $\triangle \mathrm{CDSS}$ & Baseline - follow-up & 9 & 497.4 & 0.30954 \\
\hline \multirow[t]{7}{*}{ Schizophrenia only } & $-\mathrm{GAF}$ & Baseline & 27 & 300.3 & 0.21538 \\
\hline & ${ }^{\mathrm{b}} \mathrm{CDSS}$ & Baseline & 29 & 1746.1 & 0.00217 \\
\hline & ${ }^{\mathrm{a}} \mathrm{CDSS}$ & Follow-up & 27 & -3739.3 & 0.00009 \\
\hline & PANSS excitement & Baseline & 29 & 206.5 & 0.39645 \\
\hline & aPANSS depression/anxiety & Follow-up & 29 & -1286.2 & 0.00002 \\
\hline & $\triangle$ PANSS excitement & Baseline - follow-up & 29 & 324.9 & 0.19858 \\
\hline & ${ }^{\mathrm{a}} \triangle \mathrm{CDSS}$ & Baseline - follow-up & 27 & 1358.7 & 0.00002 \\
\hline
\end{tabular}

asignificant $p$-values with Bonferroni correction

${ }^{\mathrm{b}}$ Significant $p$-values without Bonferroni correction

affective versus affective psychosis) have different PRS, whereas our sample included only non-affective FEP. Additionally, we did not observe overall PRS differences between our FEP subgroups. In addition, a recent study, within a large population cohort of adolescents, found an association between PRS and negative symptoms but not with depressive symptoms ${ }^{16}$.

One strength of our study is that all patients were antipsychotic-naive at the baseline and received the same treatment for approximately the same time. Our study also has several limitations, primary amongst which is that our FEP sample size is small $(N=60)$. The treatment used, risperidone, has been shown to be beneficial as an augmentation therapy in MDD patients who have a highrisk for suicide ${ }^{23}$ and in patients who were treatmentrefractory for $\mathrm{MDD}^{24}$. However, it is a unique longitudinal sample of antipsychotic naïve FEP individuals. We will increase this sample in the future but at the moment it represents a (near) unique resource. Despite these limitations, this is the first study to explore PRS before standardized treatment initiation in the FEP as well as the change in symptoms after a sufficient time period in which to observe response to treatment.

Our results suggest that drugs and other treatments may confound our understanding of the influence of PRS on symptomatology due to their effects on specific symptoms. We expect that future studies will explore additional clinical dimensions, taking into account the response to different antipsychotics, while increasing the sample size of treatment naïve patients analysed to have more statistical power. In conclusion, we have shown that that excitement and depressive symptoms are positively associated to SCZ-PRS during FEP pre-treatment but not after risperidone treatment and that increased SCZ-PRS is associated with the risperidone induced improvement of both depressive and excitement symptoms.

\section{Disclaimer}

The views expressed are those of the author(s) and not necessarily those of the UK NHS, the UK NIHR or the UK Department of Health. Results reflect the author's view and the Agency is not responsible for any use that may be made of the information it contains.

\section{Acknowledgements}

The authors would like to thank the patients, their families, the psychiatrists and nurses for their participation in this study. This study was supported by Fundação de Amparo à Pesquisa do Estado de São Paulo (FAPESP 2010/089686; 2011/50740-5; 2014/50830-2, 2014/07280-1; 2014/22223-4; 2012/12686-1,

2016/13737-0, 2016/04983-7), Brazil. This work was also directly supported by an UK Medical Research Council/FAPESP Newton Award (MRC MR/M026337/ 1). This work represents work supported in part by the NIHR Biomedical Research Centre ('BRC') hosted at King's College London and South London and Maudsley NHS Foundation Trust and funded by the National Institute for Health Research under its Biomedical Research Centres funding initiative. This study presents independent research supported by the Institute of Psychiatry Psychology and Neuroscience (IoPPN) Genomics \& Biomarker Core Facility within King's College London. Further information can be found at http:// www.kcl.ac.uk/ioppn/depts/mrc/research/The-loPPN-Genomics--BiomarkerCore-Facility.aspx We gratefully acknowledge capital equipment funding from the Maudsley Charity (Grant Ref. 980) and Guy's and St Thomas's Charity (Grant Ref. STR130505). 


\section{Author details}

${ }^{1}$ Genetics Division, Federal University of São Paulo (UNIFESP), São Paulo, SP, Brazil. ${ }^{2}$ Interdisciplinary Laboratory of Clinical Neurosciences, Federal University of São Paulo (UNIFESP), São Paulo, SP, Brazil. ${ }^{3}$ Social, Genetics \& Developmental Psychiatry Centre (SGDP), Institute of Psychiatry, Psychology and Neuroscience at King's College London, London, UK. ${ }^{4}$ Department of Psychiatry, Federal University of São Paulo (UNIFESP), São Paulo, SP, Brazil. ${ }^{5}$ Centro de Atendimento Integrado em Saúde Mental (CAISM), Santa Casa de Misericórdia de São Paulo, São Paulo, SP, Brazil. ${ }^{6} \mathrm{NIHR}$ Maudsley Biomedical Research Centre, South London and Maudsley NHS Trust \& King's College London, London SE5 8AF, UK

\section{Conflict of interest}

R.A.B. reports grants and non-financial support from Fundação de Amparo a Pesquisa de São Paulo (FAPESP/2016/02246-5), Brasil; grants and non-financial support from Conselho Nacional de Desenvolvimento Científico e Tecnologia (CNPq), Brasil, personal fees and non-financial support from Coordenação de Aperfeiçoamento de Pessoal de Nível Superior (CAPES), Brasil, during the conduct of the study; grants, personal fees and non-financial support from Janssen, personal fees from Ache Laboratórios Farmacêuticos, grants and personal fees from Roche, outside the submitted work. S.D.J. funding: S.D.J. was part supported by NARSAD Young Investigator Grant (YI 60373) and European Union's Horizon 2020 research and innovation programme (Marie Sklodowska-Curie grant agreement 658195). The other authors have nothing to disclose.

\section{Publisher's note}

Springer Nature remains neutral with regard to jurisdictional claims in published maps and institutional affiliations.

Supplementary Information accompanies this paper at (https://doi.org/ 10.1038/s41398-018-0230-7).

Received: 19 September 2017 Revised: 4 July 2018 Accepted: 14 July 2018 Published online: 31 August 2018

\section{References}

1. Birchwood, M., Todd, P. \& Jackson, C. Early intervention in psychosis. The critical period hypothesis. Br. J. Psychiatry Suppl. 172, 53-59 (1998).

2. Demjaha, A., MacCabe, J. H. \& Murray, R. M. How genes and environmental factors determine the different neurodevelopmental trajectories of schizophrenia and bipolar disorder. Schizophr. Bull. 38, 209-214 (2012).

3. Fuste, M. et al. Reduced expression of SP1 and SP4 transcription factors in peripheral blood mononuclear cells in first-episode psychosis. J. Psychiatr. Res 47, 1608-1614 (2013).

4. Sullivan, P. F., Kendler, K. S. \& Neale, M. C. Schizophrenia as a complex trait: evidence from a meta-analysis of twin studies. Arch. Gen. Psychiatry 60 1187-1192 (2003).
5. Schizophrenia Working Group of the Psychiatric Genomics Consortium. Biological insights from 108 schizophrenia-associated genetic loci. Nature 511, 421-427 (2014).

6. Gottesman, I. I. \& Shields, J. A polygenic theory of schizophrenia. Proc. Natl. Acad. Sci. USA 58, 199-205 (1967).

7. Wray, N. R., Goddard, M. E. \& Visscher, P. M. Prediction of individual genetic risk to disease from genome-wide association studies. Genome Res 17, 1520-1528 (2007).

8. Purcell, S. M. et al. Common polygenic variation contributes to risk of schizophrenia and bipolar disorder. Nature 460, 748-752 (2009).

9. Dudbridge, F. Power and predictive accuracy of polygenic risk scores. PLoS Genet 9, e1003348 (2013).

10. Derks, E. M., Vorstman, J. A., Ripke, S., Kahn, R. S. \& Ophoff, R. A. Investigation of the genetic association between quantitative measures of psychosis and schizophrenia: a polygenic risk score analysis. PLoS One 7, e37852 (2012).

11. Walton, E. et al. Cumulative genetic risk and prefrontal activity in patients with schizophrenia. Schizophr. Bull. 39, 703-711 (2013).

12. Whalley, H. C. et al. The influence of polygenic risk for bipolar disorder on neural activation assessed using fMRl. Transl. Psychiatry 2, e130 (2012).

13. Whalley, H. C. et al. Polygenic risk and white matter integrity in individuals at high risk of mood disorder. Biol. Psychiatry 74, 280-286 (2013).

14. Holmes, A. J. et al. Individual differences in amygdala-medial prefrontal anatomy link negative affect, impaired social functioning, and polygenic depression risk. J. Neurosci. 32, 18087-18100 (2012).

15. Vassos, E. et al. An examination of polygenic score risk prediction in individuals with first-episode psychosis. Biol. Psychiatry 81, 470-477 (2017).

16. Jones, H. J. et al. Phenotypic manifestation of genetic risk for schizophrenia during adolescence in the general population. JAMA Psychiatry 73, 221-228 (2016).

17. Sengupta, S. M. et al. Polygenic risk score associated with specific symptom dimensions in first-episode psychosis. Schizophr. Res 184, 116-121 (2017).

18. Lima, M. S. et al. The Portuguese version of the clinical global impressionschizophrenia scale: validation study. Rev. Bras. Psiquiatr 29, 246-249 (2007).

19. Bressan, R. A.r Chaves, A. C., Shirakawa, I. \& de Mari, J. Validity study of the Brazilian version of the calgary depression scale for schizophrenia. Schizophr. Res 32, 41-49 (1998).

20. Wallwork, R. S., Fortgang, R., Hashimoto, R., Weinberger, D. R. \& Dickinson, D. Searching for a consensus five-factor model of the positive and negative syndrome scale for schizophrenia. Schizophr. Res 137, 246-250 (2012).

21. Higuchi, C. H. et al. Factor structure of the positive and negative syndrome scale (PANSS) in Brazil: convergent validation of the Brazilian version. Rev. Bras. Psiquiatr 36, 336-339 (2014)

22. Leucht, S., Davis, J. M., Engel, R. R., Kissling, W. \& Kane, J. M. Definitions of response and remission in schizophrenia: recommendations for their use and their presentation. Acta Psychiatr. Scand. Suppl. 438, 7-14 (2009).

23. Reeves, $\mathrm{H}$. et al. Efficacy of risperidone augmentation to antidepressants in the management of suicidality in major depressive disorder: a randomized, double-blind, placebo-controlled pilot study. J. Clin. Psychiatry 69, 1228-1336 (2008).

24. Mahmoud, R. A. et al. Risperidone for treatment-refractory major depressive disorder: a randomized trial. Ann. Intern Med 147, 593-602 (2007). 